\title{
INTERIOR FOURIER NEAR-FIELD ACOUSTICAL HOLOGRAPHY USING ENERGY DENSITY
}

\author{
Zachary Collins, Jonathan Blotter, Kent Gee, Scott Sommerfeldt \\ Department of Mechanical Engineering, Brigham Young University
}

\begin{abstract}
Near-field acoustical holography (NAH) is used to reconstruct three-dimensional acoustic fields from a two-dimensional planar measurement. During previous work at BYU, a method has been developed called energy-based near-field acoustical holography (ENAH), which reduced the number of needed measurements by $75 \%$. Other recent advances have expanded the theory to interior spaces where multiple sources and/or reflections are present. This paper presents a new method for reconstructing interior acoustic parameters using Fourier NAH and a single plane of energy density measurements. Energy density is measured using a six-microphone array. First, the probe measurements are used to create a Hermite surface pressure interpolation on two separate planes. These two planes are used to approximate the normal particle velocity as well as to separate the incoming and outgoing waves using the spatial Fourier-transform method. Once separated, traditional Fourier NAH is used to reconstruct the pressure and normal particle velocity at any point in space. Analytical and experimental results are shown and compared to exterior Fourier NAH approximations. Other drawbacks and benefits are discussed.
\end{abstract}

\section{INTRODUCTION}

In order to identify and characterize noise sources, near-field acoustical holography (NAH) is a technique that uses a two-dimensional measurement to reconstruct a three-dimensional acoustic field. Assumptions for the conventional NAH method limit its use to exterior fields where a single noise source is propagating. Many practical situations where noise source identification would be advantageous take-place in an enclosed space where reflections are present, this is where conventional NAH fails to be useful.

The objective of this research is to develop a noninvasive measurement technique to measure a sound field inside an enclosure. The technique needs to be able to characterize a sound source inside the enclosure by separating reflected wave components from the actual noise source, then create an accurate reconstruction of the source. This technique is to be an inexpensive alternative to using a Scanning Laser Doppler Vibrometer (SLDV), as well as reduce the number of measurements needed by utilizing all the information available in an energy-based measurement.

\section{BACKGROUND}

Imaging techniques in acoustics have been useful in determining the behavior of vibrating structures. These techniques are commonly used in numerous applications to identify and characterize noise sources and energy distributions on source surfaces. Near-field acoustical holography is one such imaging technique in which acoustical measurements made along a two-dimensional surface can be used to calculate both 3-D acoustic quantities and the vibration properties of the radiating structure.

Past developments of NAH techniques involve a common assumption, that when acoustic pressure measurements are made along a two-dimensional plane, all sources lie on one side of that plane. This assumption makes these techniques valid for exterior sound fields, or fields where reflection is not an issue. When trying to reconstruct an interior noise source, a technique is needed to distinguish 
between the incoming and outgoing waves. Expanding NAH to include interior spaces has been the focus of recent research in the acoustics community.

Williams ${ }^{1}$ demonstrated how a scanning boom that takes pressure measurements inside an enclosure can be used to inspect the surfaces of the enclosure. Langrenne ${ }^{2}$, Jacobsen ${ }^{3}, \mathrm{Hald}^{4}$, and $\mathrm{Bi}^{5}$ have recently developed techniques for separating the incoming and outgoing waves. They all used two closely-spaced parallel arrays of pressure measurements and have reconstructed the acoustic field using different methods, including statistically optimized near-field acoustical holography (SONAH), the inverse-boundary element method (IBEM), and an equivalent source method. Jacobsen ${ }^{6}$ also determined that using a single layer pressure-velocity measurement was equivalent to using a double layer pressure measurement. $\mathrm{Yu}^{7}$ has shown that measuring a field's acoustic pressure gradient along with its simultaneous, co-located acoustic pressure, then solving for the three-dimensional field using an Acoustic Intensity-Based method improves the accuracy, reliability, and consistency of reconstructed acoustic radiation pressure. It also increases random noise tolerance from $1 \%$ to $20 \%$.

A unifying drawback for all of the currently published methods is that they all require a large amount of time and/or computation. Most require custom microphone arrays and all of these methods could benefit from lowering the number of measurements required. Reconstructing an acoustic hologram accurately using all of these methods requires an adequate and precise representation of the pressure field on the measurement surface. Both the Fourier transform $\operatorname{method}^{8}$ and the IBEM rely on special sampling for field characterization, which becomes cumbersome due to the fact that the microphone spacing must be less than or equal to a half wavelength of the highest frequency of interest to avoid spatial aliasing.

\section{METHOD}

For ease in computation, the Fourier NAH method has been selected for displaying the benefits of using an energy-based measurement method for

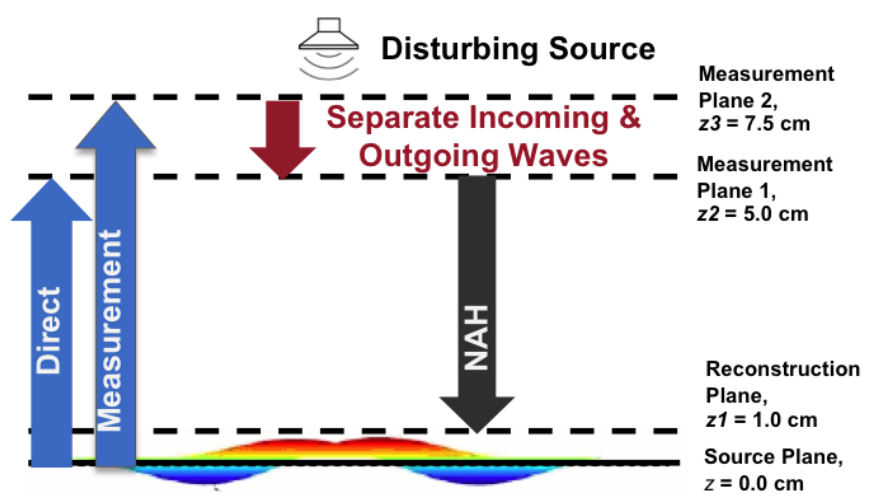

Figure 1: A diagram of the process to find the acoustic properties of a source by making a measurement in a reverberant field. A direct pressure measurement is made on two separate planes, the incoming and outgoing waves are separated, then NAH is performed to propagate back to the source.

interior NAH. The Fourier technique is beneficial for sources that comply with Cartesian coordinates: rectangular rooms, cylindrical rooms, or spherical rooms. These orthogonal coordinate frames work well with the computational speed that Fourier NAH offers.

To perform NAH on an interior space using the Fourier method, six steps are taken (a summary of the steps is shown in Figure 1). First, sound pressure measurements are taken on two separate planes parallel to the source in the near-field (planes $\mathrm{z}_{2}$ and $\mathrm{z}_{3}$ shown in Figure 1). Second, the sound field is interpolated using the additional data from a six-microphone probe. This measurement and interpolation technique is discussed in part A. Third, a two-dimensional spatial Fourier transform is performed on the two separate planes, breaking the field up into the contributing wave-numbers. Fourth, the incoming and outgoing waves are separated using the Spatial Fourier Transform Separation Technique ${ }^{9}$ (discussed in part B). Fifth, traditional Fourier $\mathrm{NAH}^{8}$ is performed on only the outgoing portion of the waves to obtain an estimation of the wavenumbers on a plane next to the source (plane $\mathrm{z}_{1}$ shown in Figure 1). Finally, an inverse twodimensional spatial Fourier Transform is performed to find the complex acoustic pressure or particle velocity of the source.

Analytical experiments and a small number of physical experiments have been performed to validate this method. 


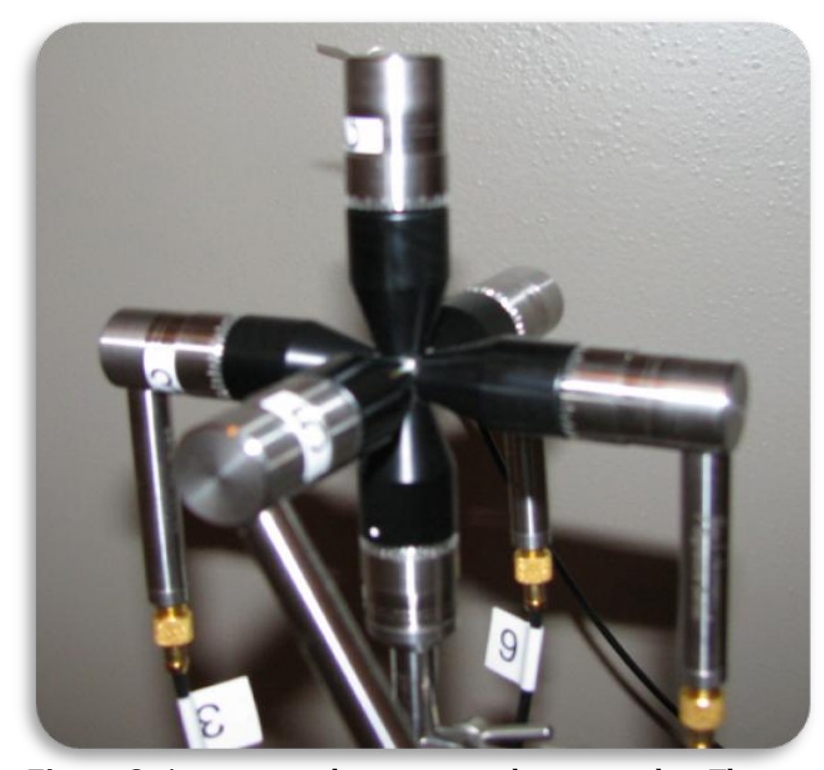

Figure 2: A six-microphone energy density probe. This probe enables two plane's of measurements to be made.

\section{A. Using a Six-Microphone Probe to Interpolate a Pressure Field}

To perform a source separation, two planes of measurements must be made. This paper proposes using a six-microphone intensity probe (as shown in Figure 2) to make the measurements. The use of a probe like this has two main benefits. First, it allows the pressure to be measured on two separate planes (as shown by microphones 1 and 2 in Figure 3), and second, the particle velocity can be estimated by the four remaining microphones between the two planes. Utilizing the information from all six microphones will allow interpolation of the pressure field so spatially courser measurement can be made.

This measurement technique is referred to as "energy-based" because it uses both the pressure and the particle velocity. Energy density is the combination of kinetic and potential energy respectively comprised of a squared particle velocity component and a squared pressure

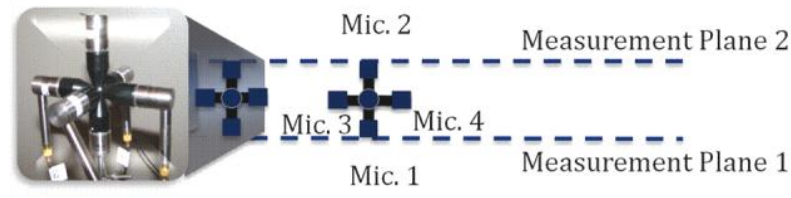

Figure 3: The six-microphone probe allows pressure measurements to be made simultaneously on two separate planes, while the four microphones between the planes are used to calculate the pressure gradient. component.

Even though energy density won't be measured or calculated directly, the technique presented is considered an energy-based approach because of it's reliance on pressure and particle velocity.

As stated previously, microphones 1 and 2 are used to measure the pressure simultaneously on two separate planes. The benefit of using four microphones between the two planes is that an estimate of the pressure gradient for the two inplane directions can be estimated. These pressure gradients on the mid-plane can be used to estimate the pressure gradients on measurement planes 1 and 2. The probe is moved around until an adequate grid of measurements has been taken.

After the measurement process is finished, the result is a rectangular grid of pressure measurements with corresponding collocated inplane pressure gradients. After these values are known on both parallel planes, the pressure can be interpolated using the collocated pressure gradients.

The interpolation is performed using a Hermite patch interpolation technique described by Harris ${ }^{10}$. This technique uses the pressure and it's slope to interpolate between measurement locations. Harris showed that by using this interpolation technique, the number of required measurement locations can be reduced by as much as $75 \%$. The reduction he calculated was achieved by just using four microphones to interpolate one plane. So far in this research, the measurement location reduction of $75 \%$ has proven to be likely as well.

\section{B. The Spatial Fourier Transform Separation Technique}

Another benefit of using the Fourier NAH method is that it can easily implement a separation technique that involves a Spatial Fourier Transform. Tamura ${ }^{9}$ recently showed how to measure reflection coefficients by taking two parallel planes of measurements and working in the wave-number domain. The incident and reflected waves can be separated out by using the following formulas adapted from Tamura' 


$$
\begin{aligned}
P_{i}\left(k_{x}, k_{y}, z_{2}\right)= & \frac{P\left(k_{x}, k_{y}, z_{1}\right)-P\left(k_{x}, k_{y}, z_{3}\right) e^{i k_{z}\left(z_{3}-z_{2}\right)} e^{-i k_{z}\left(z_{1}-z_{2}\right)}}{e^{i k_{z}\left(z_{1}-z_{2}\right)}-e^{2 i k_{z}\left(z_{3}-z_{2}\right)} e^{-i k_{z}\left(z_{1}-z_{2}\right)}} \\
P_{r}\left(k_{x}, k_{y}, z_{2}\right)= & P\left(k_{x}, k_{y}, z_{3}\right) e^{i k_{z}\left(z_{3}-z_{2}\right)}- \\
& {\left[\frac{P\left(k_{x}, k_{y}, z_{1}\right)-P\left(k_{x}, k_{y}, z_{3}\right) e^{i k_{z}\left(z_{3}-z_{2}\right)} e^{-i k_{z}\left(z_{1}-z_{2}\right)}}{e^{i k_{z}\left(z_{1}-z_{2}\right)}-e^{2 i k_{z}\left(z_{3}-z_{2}\right)} e^{-i k_{z}\left(z_{1}-z_{2}\right)}}\right] e^{2 i k_{z}\left(z_{3}-z_{2}\right)} }
\end{aligned}
$$

where $P$ is the complex transformed pressure, $k_{x}$, $k_{y}$, and $k_{z}$ are the wave-numbers in the $\mathrm{x}, \mathrm{y}$, and $\mathrm{z}$ directions respectively, $z_{2}$ is the measurement plane closest to the source, and $z_{3}$ is the measurement plane parallel to $z_{2}$, but further from the source. The two above equations will lead to the separated incident and reflected pressure components.

\section{RESULTS}

At this point in the research, analytical simulation has been used to prove that this technique will work, while experimental verification is still occurring.

The first question that needed to be answered was if the pressure gradients measured between the two measurement planes could be used to approximate the actual pressure gradients. Figure 4 shows the error which results from using a separate plane's pressure gradient as an

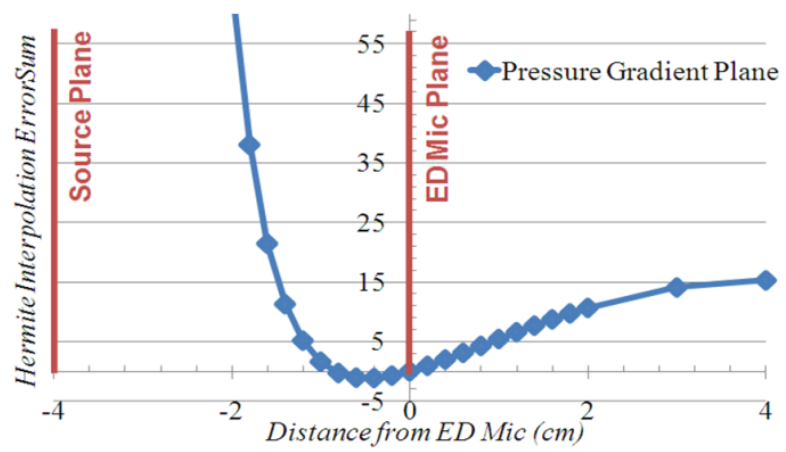

Figure 4: The error associated with using a separate plane's pressure gradient as an approximation. The pressure gradient was approximated on a plane that varied from two cm closer to the source to four $\mathrm{cm}$ further away from the source. As can be seen, when the gradient measurement is approximated on a plane close to the actual plane, very little error is introduced. The test frequency shown here is $300 \mathrm{~Hz}$. approximation of another plane. The errors introduced by this approximation are very small as long as the approximation is made close to the measurement plane.

A simulation was also run to see if the separation method was beneficial when there was a reflection present. For this simulation, the noise source consisted of a set of four point sources radiating at $300 \mathrm{~Hz}$. A reflective wall caused a reflection back to the measurement plane. This wall location was varied from 4 to $30 \mathrm{~cm}$. The measurements plane was $4 \mathrm{~cm}$ away from the source. Three different cases were investigated. Figure 5 shows the results of this simulation. First, the error was found using the original free-field $\mathrm{NAH}$ code which assumes there are no reflections or additional sources. The error was very large when the wall reflection was comparable to the direct pressure contribution. The separation technique described above was also used in the simulation, the error associated with this technique was about $0.2 \%$ of error without any separation when the wall was located next to the measurement plane. The spatial Fourier transform separation technique was still beneficial regardless of the reflective wall location.

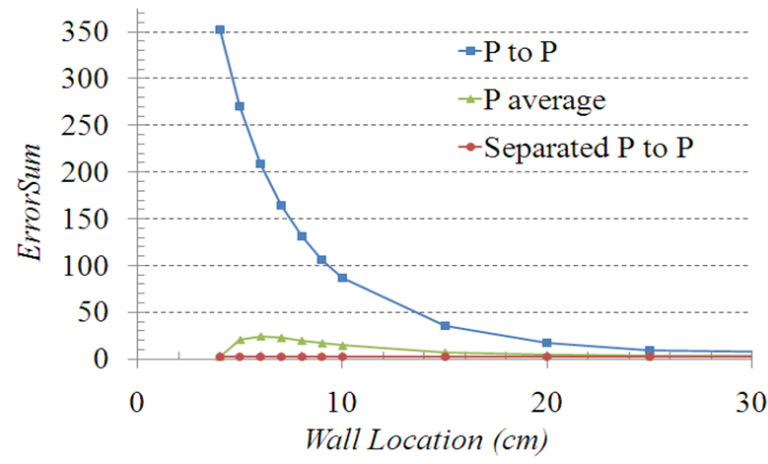

Figure 5: The error associated with performing $\mathrm{NAH}$ when a reflection is present. The reflective wall is varied from 4 to $30 \mathrm{~cm}$. Free-field NAH shown in blue shows a large error when the reflective wall is close to the measurement plane. When the spatial Fourier transform technique is used before $\mathrm{NAH}$, shown in red, there is minimal error in the NAH reconstruction regardless of how strong the reflection is. An additional separation technique not described in this paper is shown in green. 
Physical experimentation has been started to validate this measurement technique. So far, measurements made with no reflection present have been successfully propagated back to the source using a six-microphone probe. Further testing will soon be done to validate the technique when there are one or more reflections or sources present.

Further testing will also be done to determine the reduction in measurement locations achieved when using the six-microphone interpolation technique.

\section{CONCLUSIONS}

By making measurements with a six-microphone probe, both the pressure and in-plane particle velocity can be estimated.

The four mid-plane microphones can be used to estimate the pressure gradient on the adjoining planes without accumulating too much additional error to the measurement.

The estimated pressure gradient can be used for a Hermite surface patch interpolation that will reduce the number of required measurement locations.

The interpolated pressure on two measurement planes can be used to separate incident and reflected waves.

The success of this measurement method seems to depend on frequency as well as the strength of disturbing sources or reflections.

\section{REFERENCES}

[1] E.G. Williams, B. H. Houston, P. C. Herdic, S. T. Raveendra, and B. Gardner, "Interior near-field acoustical holography in flight," J. Acoust. Soc. Am. 108, 1451-1463 (2000).

[2] C. Langrenne, M. Melon, and A. Garcia, "Boundary element method for the acoustic characterization of a machine in bounded noisy environment," J. Acoust. Soc. Am. 121, 2750-2757 (2007).
[3] F. Jacobsen and V. Jaud, "Statistically optimized near field acoustic holography using an array of pressure-velocity probes,” J. Acoust. Soc. Am. 121, 1550-1558 (2007).

[4] J. Hald, C. Blaabjerg, M. Kimura, Y. Ishii, M. Tsuchiya, H. Ando, and T. Yamashita, "Panel contribution analysis using a volume velocity source and a double layer array with the SONAH algorithm," Proceedings of Inter-Noise 2006, Honolulu, HI, Dec 36, 2006, (on CD) Paper in06_167, ,10 pages.

[5] C. X. Bi, X. Z. Chen, and J. Chen, "Sound field separation technique based on equivalent source method and its application in nearfield acoustic holography," J. Acoust. Soc. Am. 123, 1472-1478 (2008).

[6] F. Jacobsen, X. Chen, and V. Jaud, "A comparison of statistically optimized near field acoustic holography using single layer pressure-velocity measurements and using double layer pressure measurements," J. Acoust. Soc. Am. 123, 1842-1845 (2008).

[7] C. Yu, Z. Zhou, and M. Zhuang, "An acoustic intensity-based method for reconstruction of radiated fields," J. Acoust. Soc. Am. 123, 1892-1901 (2008).

[8] E.G. Williams, Fourier Acoustics: Sound Radiation and Nearfield Acoustical Holography. (Academic Press, San Diego, CA 1999).

[9] M. Tamura, "Spatial Fourier transform method of measuring reflection coefficients at oblique incidence. I: Theory and numerical examples," J. Acoust. Soc. Am. 88, 2259-2264 (1990).

[10] M. C. Harris, J. D. Blotter, and S. D. Sommerfeldt, "Obtaining the complex pressure field at the hologram surface for use in near-field acoustical holography when pressure and in-plane velocities are measured," J. Acoust. Soc. Am. 119, 808-816 (2006). 